\title{
The Effect of $\mathrm{NaCl} / \mathrm{pH}$ on Colloidal Nanogold Produced by Pulsed Spark Discharge
}

\author{
Kuo-Hsiung Tseng, Chin-Liang Hsieh, Jen-Chuen Huang, and Der-Chi Tien \\ Department of Electrical Engineering, National Taipei University of Technology, Taipei 10608, Taiwan \\ Correspondence should be addressed to Kuo-Hsiung Tseng; khtseng@ee.ntut.edu.tw
}

Received 20 May 2015; Revised 8 August 2015; Accepted 16 August 2015

Academic Editor: Themis Matsoukas

Copyright ( $) 2015$ Kuo-Hsiung Tseng et al. This is an open access article distributed under the Creative Commons Attribution License, which permits unrestricted use, distribution, and reproduction in any medium, provided the original work is properly cited.

\begin{abstract}
A green method, using pulsed spark discharge (PSD) to synthesize colloidal gold, is studied in this thesis. PSD uses spark discharge to synthesize gold nanoparticles (AuNPs) in deionized water (DIW) and/or ethanol (EtOH). While gold nanoparticles have widespread applications in many fields, especially for the human body, in use them must overcome the influence of $\mathrm{NaCl}$ and $\mathrm{pH}$ value; therefore, this study adds $\mathrm{NaCl}$ into PSD-AuNPs to simulate the human body to study its stability. Furthermore, a variety of protectants are added in an attempt to determine the best protectant for AuNPs and improve biologically compatible potency. From the results of this study, adding the long-chain-polymer Carboxymethyl cellulose (CMC) or Polyvinyl pyrrolidone (PVP-k30) can prevent nanogold from aggregation and precipitation in $\mathrm{NaCl}$ or different $\mathrm{pH}$ value and maintain the characteristic of nanogold dispersion by raising the repulsive force between the particles. The results of this study can be a reference of nanogold applying in biomedical science.
\end{abstract}

\section{Introduction}

The pulse spark discharge (PSD) method is developed and used to fabricate the AuNPs solution [1-5], which involves a pulse current being passed through two gold electrodes [68 ], which are submerged in deionized water or ethanol. Many methods of producing AuNPs include the introduction of surfactants in order to improve the suspension of the gold particles. However, gold nanoparticles fabricated by the pulse spark discharge (PSD) method in deionized water or ethanol without any surfactants or stabilizers are characterized as a stable colloid, which can be stored for a long term in a glass container at room temperature without visible sedimentation (no apparent precipitate). The DIW_nanogold is safe for the human body, such as target therapy and drug carriers; this study will propose the experiments and simulate results of the colloidal gold [9-13] within the $\mathrm{NaCl}$ and $\mathrm{pH}$ test.

Gold nanoparticles are widely applied in the human body but require overcoming the impacts of $\mathrm{NaCl}$ and $\mathrm{pH}$ value. Gold number [14] is defined as the amount (mg) of polymer required to prevent the aggregation of $10 \mathrm{~cm}^{3}$ of gold solution with $1 \mathrm{~cm}^{3} 10 \% \mathrm{NaCl}$ added. This study also proposes an effective gold number method (a more efficient version of Zsigmondy's [15] method is conducted in the experiment performed here) in order to determine the gold number of potential colloidal gold stabilizers. Instead of varying the amount of surfactant added, increasing amounts of $\mathrm{NaCl}$ are added into a solution of colloidal gold with $0.1 \mathrm{mg}$ of stabilizer, thus simulating continuous titrations. Also, the photothermal effect of AuNPs can also be used as a cancer treatment [16]. The superior biological piezoelectric biosensors can be produced, through biocompatibility, the electrical conductivity and the high surface area of nanogold particles [17]. The properties of nanoparticles can apply to metal to produce beneficial reaction of catalytic [18]. This study simulates the human body or normal saline in $\mathrm{NaCl}$ in order to discuss the impacts on gold nanoparticles of DIW_nanogold, under various biologically compatible protective agents, for improving biologically compatible potency. The impacts on, and variations in, suspension of $\mathrm{pH}$ value of gold nanoparticles (DIW_nanogold, chem._nanogold, and ethanol nanogold) fabricated by other methods, as well as the changes of absorbance and wavelength, are compared. 


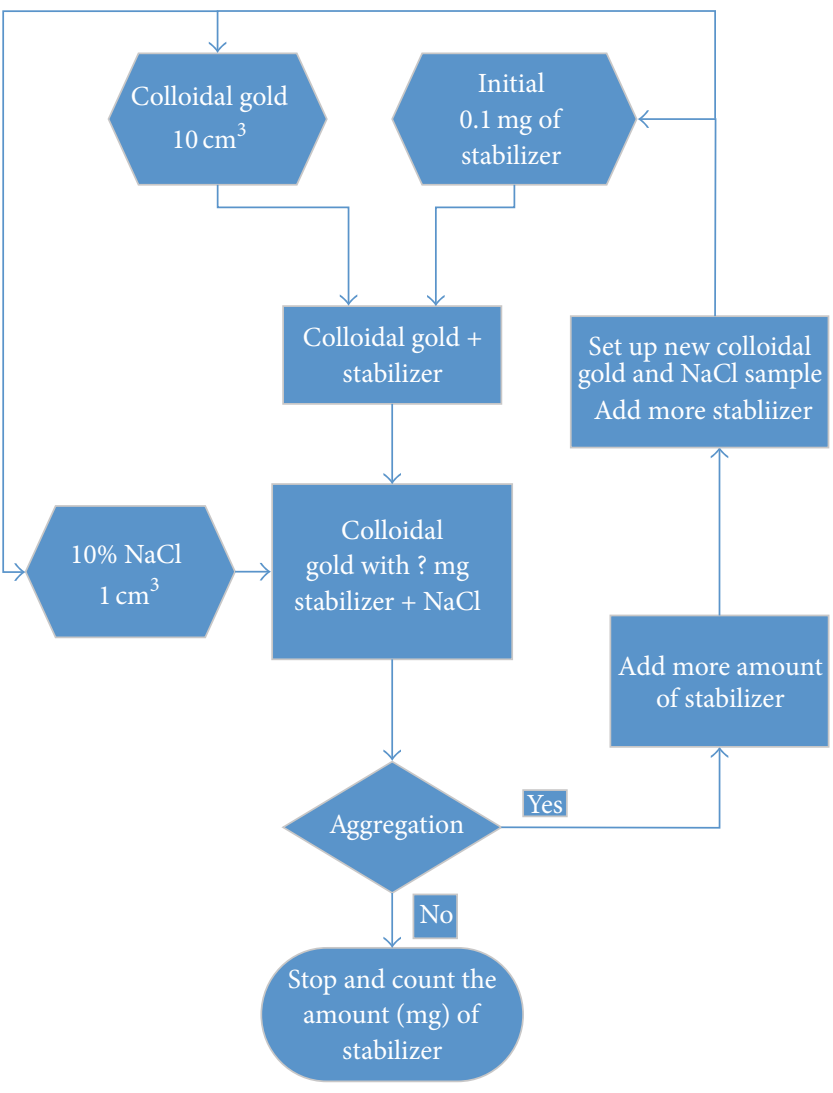

(a)

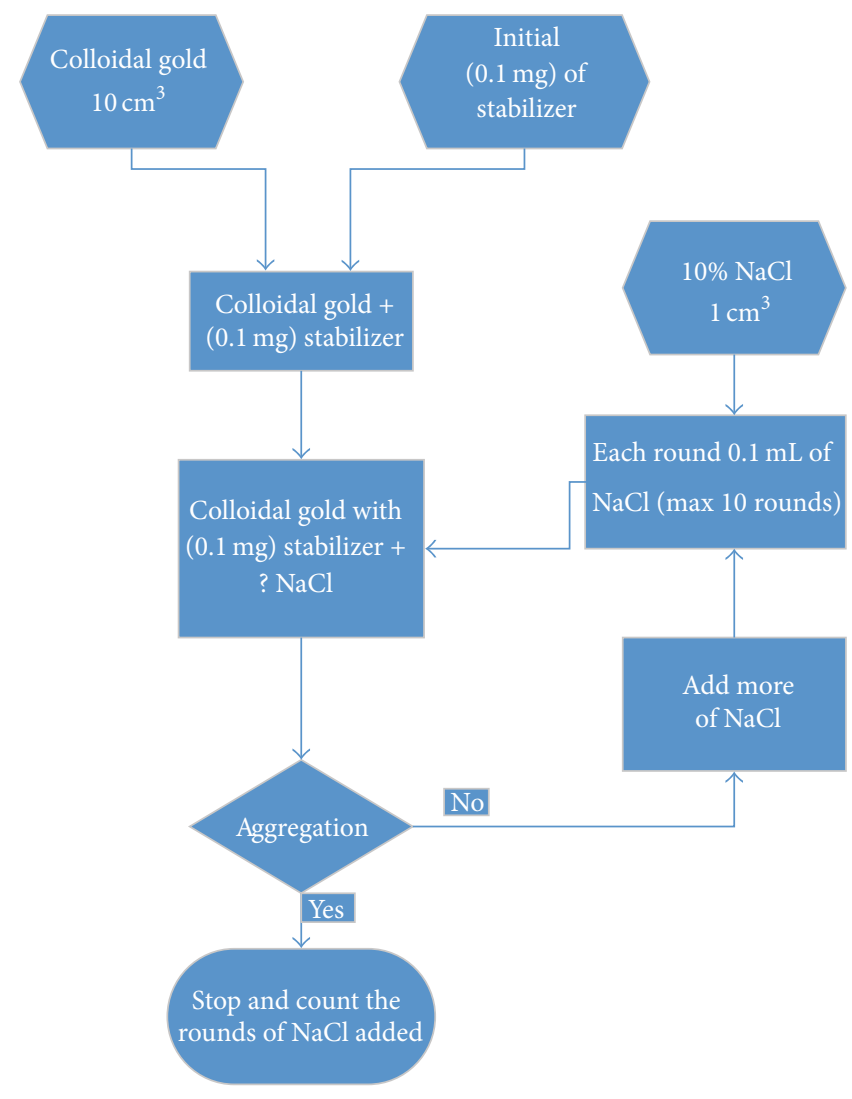

(b)

FIgURE 1: Flow chart of (a) Zsigmondy's method and (b) more efficient version of Zsigmondy's method.

\section{Experimental Setup}

This study utilizes the developed PSD system as a preparation method; the principle is to use a bar material ( $\mathrm{Au}$ ), which will be generated into nanometal material as top and bottom electrodes; there exists no direct contact between the two electrodes; thus, there is no physical force produced between the two; however, by using electricity converted into heat energy, a kind of hot melting method of electrode rapidly melting is created. The chamber is the main processing center. Deionized water, which has good insulativity, or ethanol, is used as dielectric liquid. The top and bottom electrodes are submerged into dielectric liquid to cause the generated nanoparticles to spread evenly and be directly stored in the dielectric liquid.

2.1. Preparation for $\mathrm{NaCl}$ Test. The fourteen agents listed in Table 1 were tested for their ability to maintain gold colloidal suspension. With the simulation of $\mathrm{NaCl}$ in proportions similar to those in human body fluids $(0.9 \%)$, or the proportion of $\mathrm{NaCl}$ in normal saline, colloidal gold is mixed with a highly concentrated $\mathrm{NaCl}$ solution, which results in $\mathrm{Na}^{+}$and $\mathrm{Cl}^{-}$ ions attacking the surface electric potential of the AuNPs. With the loss of their zeta potential, the nanoparticles lose their mutual repulsion and agglomerate. In order to avoid the destruction of zeta potential, the use of an adequate protecting agent is required so that the gold colloid can
TABLE 1: Types of stabilizing agents.

\begin{tabular}{lc}
\hline Item \# & Name \\
\hline$S(1)$ & Propylene glycol alginate \\
S(2) & Sodium alginate \\
S(3) & Animal glue \\
S(4) & Gelatin L-150 \\
S(5) & Guar gum S-200 \\
S(6) & Xanthan gum \\
S(7) & CMC (carboxymethyl cellulose) \\
S(8) & Pectin \\
S(9) & Carrageenan \\
S(10) & SDS (sodium dodecyl sulfate) \\
S(11) & PVP-k30 (polyvinyl pyrrolidone) \\
S(12) & Citric acid \\
S(13) & Glycine \\
S(14)
\end{tabular}

survive in such an ion-rich solution. This study analyzed the impacts of various protecting agents (must be biocompatible for use in medicinal applications) on the agglomerate of PSDAuNPs-DIW and the agent can be used as protecting agent for PSD-AuNPsDIW.

First, $10 \mathrm{~mL}$ of the $30 \mathrm{ppm}$ colloidal gold solution is placed into each of the 15 containers, numbered $\mathrm{Au}(0) \sim \mathrm{Au}(14)$; then, 


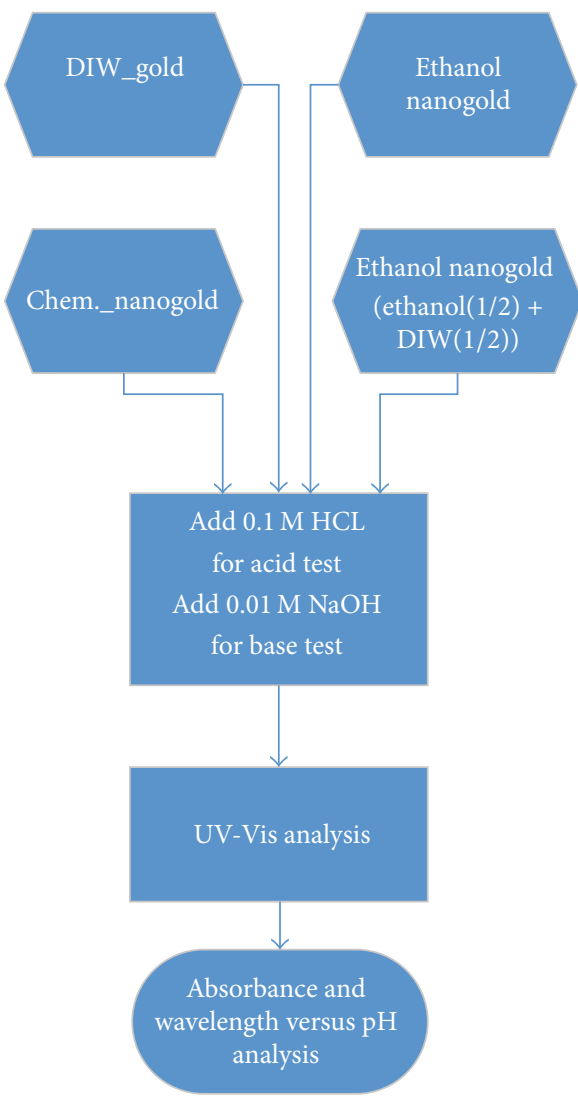

FIGURE 2: Flow chart of $\mathrm{pH}$ effect analysis for DIW_nanogold, chem._nanogold, and ethanol nanogold.

a second set is prepared by placing $10 \mathrm{mg}$ of the 14 stabilizers into $10 \mathrm{~mL}$ of $\mathrm{DIW}$ for a $0.1 \%(\mathrm{w} / \mathrm{v})$ concentration, which are labeled $\mathrm{S}(1) \sim \mathrm{S}(14)$. Then, $0.1 \mathrm{~mL}$ is taken from each of $\mathrm{S}(1) \sim \mathrm{S}(14)$ and placed into $\mathrm{Au}(1) \sim \mathrm{Au}(14)$ so that $0.1 \mathrm{mg}$ of the agent is present. No agents are added into $\mathrm{Au}(0)$, the control solution. A mixture of $1 \mathrm{~g}$ anhydrous salt, with $10 \mathrm{~mL}$ of water, is used for titrations in the amount of $0.1 \mathrm{~mL}$ (per round), and color change is noted after each titration. Add $0.1 \mathrm{~mL}$ of the $10 \% \mathrm{NaCl}$ solution in each round. After the tenth round, the proportion of $\mathrm{NaCl}(1 / 11)$ is the amount seen, on average, in human body fluids $(0.9 \%)$; therefore, the experiment can be stopped at that point. The continuous titrations will show, through level of color change, which stabilizer is the most effective. Figure 1 presents the flow chart of (a) Zsigmondy's method and (b) presents the more efficient version of Zsigmondy's method.

2.2. Preparation of $p H$ Test. For ethanol nanogold, ethanol nanogold (ethanol(1/2) + DIW(1/2)), DIW_nanogold, and chem.- nanogold, six bottles of $10 \mathrm{ml}$ of each kind are used as a sample group. Each sample group adds $\mathrm{HCl}$ for acid tests and $\mathrm{NaOH}$ for base tests, and then the change of $\mathrm{pH}$, visual observation of sample group, color changes, and agglomerate are observed. UV-Vis analysis is conducted for nonagglomerated samples and the $\mathrm{pH}$ effects on absorbance and wavenumber are analyzed, in order to gain further understanding of the relationships between the suspension of various gold nanoparticles and $\mathrm{pH}$. Figure 2 presents the flow chart of $\mathrm{pH}$ effect analysis for DIW_nanogold, chem._nanogold, and ethanol nanogold.

\section{Results and Discussion}

3.1. Results and Discussion of $\mathrm{NaCl}$ Test. Table 2 displays the number of rounds each surfactant required before a major color change was observed. Once one was observed, no more rounds of $\mathrm{NaCl}$ were added.

Reference is round 0: if color change is observed after the stabilizer is mixed with colloidal gold, but before any $\mathrm{NaCl}$ is added, round 0 is recorded. The statues are analyzed as follows:

(1) Nanogold particles which change color just after the salt solution is added: $\mathrm{Au}(6)$ and $\mathrm{Au}(10)$.

(2) Nanogold particles that aggregated, precipitated, or turned white: $\mathrm{Au}(2)$ and $\mathrm{Au}(11)$.

(3) Nanogold particles that turned blue-violet: Au(1), $\mathrm{Au}(3), \mathrm{Au}(4), \mathrm{Au}(5), \mathrm{Au}(8), \mathrm{Au}(9), \mathrm{Au}(13)$, and $\mathrm{Au}(14)$.

(4) Nanogold particles which did not agglomerate: $\mathrm{Au}(7)$ and $\mathrm{Au}(12)$.

It is therefore determined that CMC and PVP-k30 solutions provide the best protection against agglomeration in colloidal 


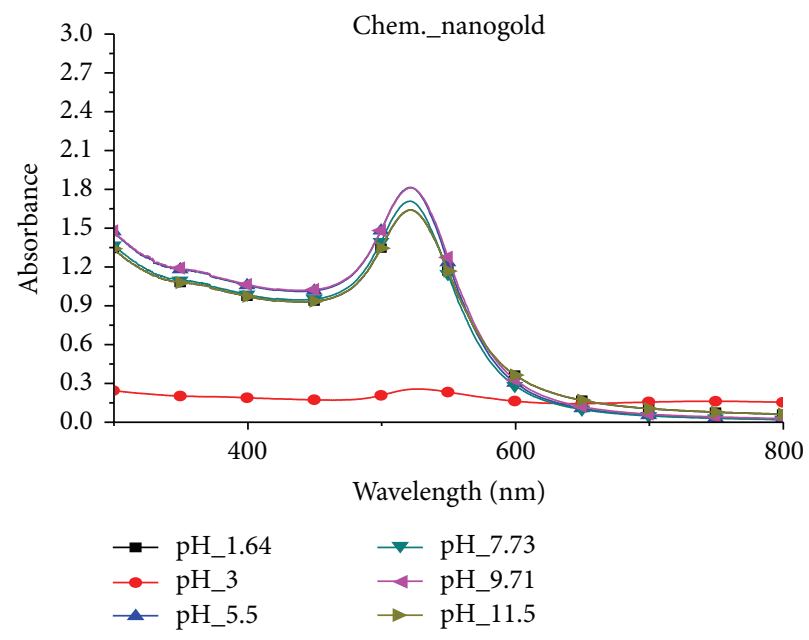

(a)

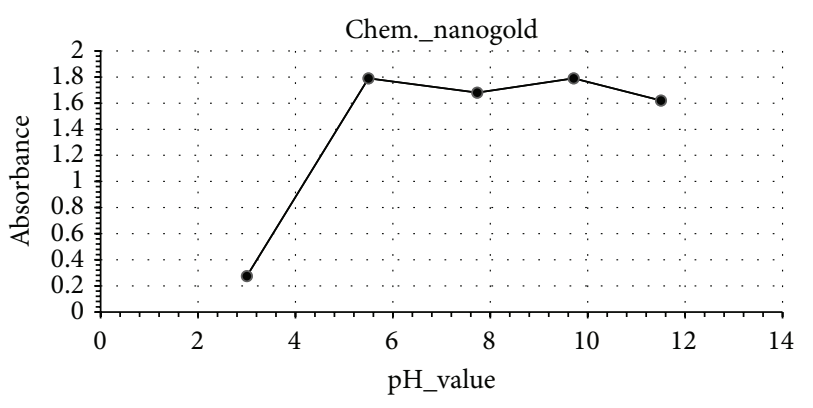

(b)

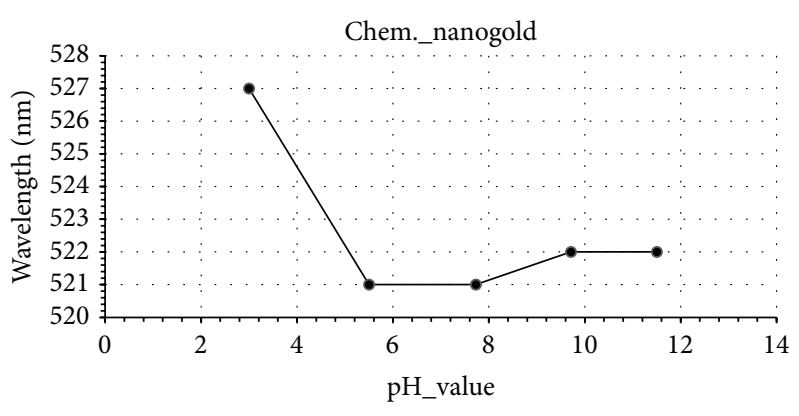

(c)

FIGURE 3: (a) SPR, (b) absorbance, and (c) wavelength versus pH of chem._nanogold.

TABLE 2: Number of $\mathrm{NaCl}$ rounds.

\begin{tabular}{lcc}
\hline Item \# & Surfactant $(0.1 \mathrm{mg})$ & $\mathrm{NaCl}\left(10^{-1} \mathrm{~mL}\right)$ \\
\hline $\mathrm{Au}(0)$ & No surfactant & 1 \\
$\mathrm{Au}(1)$ & Propylene glycol alginate & 6 \\
$\mathrm{Au}(2)$ & Sodium alginate & 1 \\
$\mathrm{Au}(3)$ & Animal glue & 3 \\
$\mathrm{Au}(4)$ & Gelatin L-150 & 5 \\
$\mathrm{Au}(5)$ & Guar gum S-200 & 8 \\
$\mathrm{Au}(6)$ & Xanthan gum & 0 \\
$\mathrm{Au}(7)$ & CMC (carboxymethyl cellulose) & 10 \\
$\mathrm{Au}(8)$ & Pectin & 7 \\
$\mathrm{Au}(9)$ & CMC (antiacid) & 7 \\
$\mathrm{Au}(10)$ & Carrageenan & 0 \\
$\mathrm{Au}(11)$ & SDS (sodium dodecyl sulfate) & 2 \\
$\mathrm{Au}(12)$ & PVP-k30 (polyvinyl pyrrolidone) & 10 \\
$\mathrm{Au}(13)$ & Citric acid & 2 \\
$\mathrm{Au}(14)$ & Glycine & 2 \\
\hline
\end{tabular}

nanogold. Of these, CMC is the safer choice, as it presents no harm to the human body and is even used in some food items to maintain a food-particle suspension.
3.2. Results and Discussion of pH Test. Chem._nanogold at $\mathrm{HCl}$ and $\mathrm{NaOH}$ condition, the relation of absorbance and wavelength with $\mathrm{pH}$, as shown in Figures 3(a), 3(b), and 3(c). AuNPs-DIW at $\mathrm{HCl}$ and $\mathrm{NaOH}$ condition, the relation of absorbance and wavelength with $\mathrm{pH}$, as shown in Figures 4(a), 4(b), and 4(c). Ethanol nanogold at $\mathrm{HCl}$ and $\mathrm{NaOH}$ condition, the relation of absorbance and wavelength with $\mathrm{pH}$, as shown in Figures 5(a), 5(b), and 5(c). Table 3 shows absorbance and wavelength deviation at different nanogold versus $\mathrm{pH}$.

In $\mathrm{pH}$ test, the higher $\mathrm{pH}$ will cause the optical property of AuNPs to have a blue shift of the peak wavelength, indicating that the suspension capacity can be improved when the gold nanoparticles are in an alkalic environment.

Table 3 shows absorption and wavelength of colloidal nanogold in different $\mathrm{pH}$ value. As shown in Figures 3(c), $4(\mathrm{c}), 5(\mathrm{c})$, and 6(c), in acid liquid, the wavelength of colloidal nanogold is longer, which is red shifting. The zeta potential is lower and also the Debye Length is shorter. These cause the nanogold particles to assemble and precipitate. In alkalic liquid, the wavelength of colloidal nanogold is shorter, which is blue shifting. The absorption of UV-Vis is higher and the zeta potential is higher. Also, the Debye Length is longer. These cause the nanogold particles to disperse. As the results show, in alkali liquid, the nanogold particles are more separated. Under the circumstances of $\mathrm{pH} 13$ and 2, the UV 


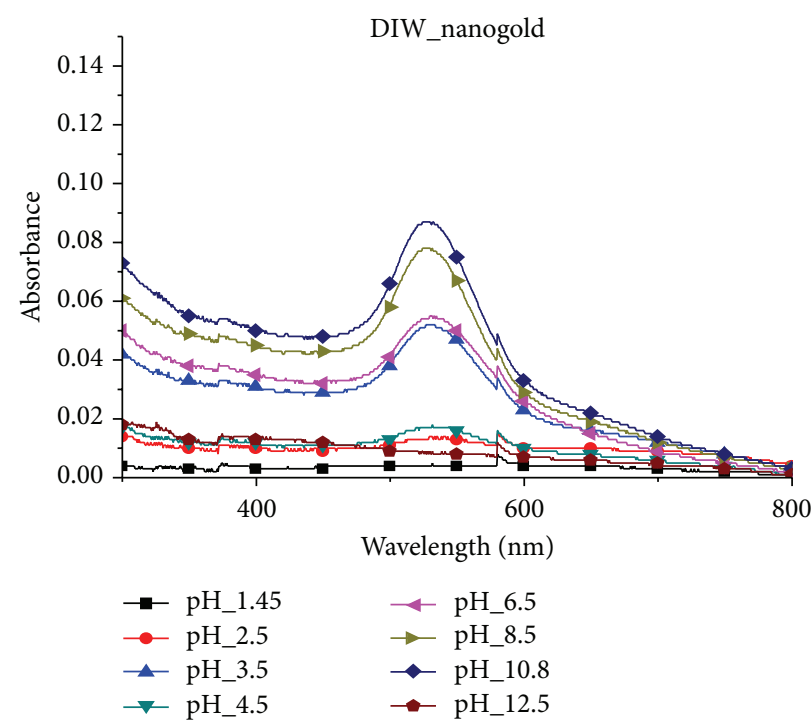

(a)

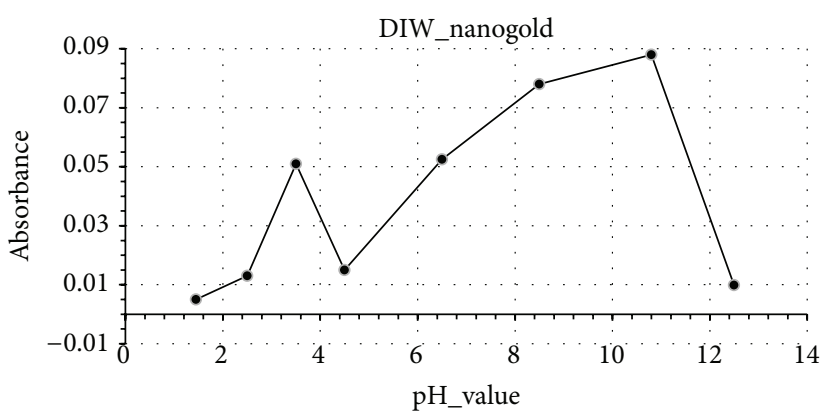

(b)

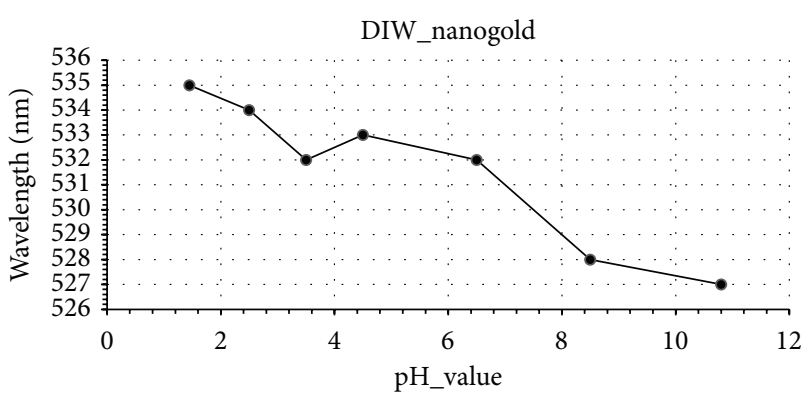

(c)

FIGURE 4: (a) SPR, (b) absorbance, and (c) wavelength versus pH of AuNPs-DIW.

absorption value of colloidal nanogold is 0 where it appears that the AuNPs drop to the bottom of the container.

\section{Conclusions}

A method of spark discharge (PSD) for producing gold nanoparticles in organic or inorganic medium (ethanol/ water) without additives is proposed. The most significant contribution of this PSD method research is the capability to directly fabricate gold nanoparticles with different particle sizes and shapes in different medium. Moreover, the gold nanoparticles produced through PSD method were welldispersed and form a stable suspension for an extended period of time. Generally, to obtain stable suspension of colloidal gold, the chemical preparation method requires posttreatments (e.g., heating, dialysis, and purification), as well as long preparation and waiting time. This PSD technique does not require complicated pretreatment or any chemical additive and can instantly achieve stable suspension of gold colloid solution.

In this study, adding or not adding different kinds of surfactant and adding different concentration of $\mathrm{NaCl}$ to see the different color changes are discussed. It is determined
TABLE 3: Absorbance and wavelength deviation at different nanogold versus $\mathrm{pH}$.

\begin{tabular}{lccl}
\hline Nanogold/item & pH value & Absorbance & Wavelength (nm) \\
\hline Chem._nanogold & $3.1 \sim 11.5$ & Increase & From 527 to 522 \\
AuNPs-DIW & $2.5 \sim 10.8$ & Increase & From 534 to 527 \\
$\begin{array}{l}\text { Ethanol nanogold } \\
\text { Ethanol nanogold }\end{array}$ & $7.1 \sim 11.5$ & Increase & From 544 to 534 \\
$\begin{array}{l}(1 / 2 \text { ethanol }+1 / 2 \\
\text { DIW })\end{array}$ & $3.1 \sim 10.5$ & No change & From 540 to 520 \\
\hline
\end{tabular}

under what condition the colloidal nanogold is better in the combination of surfactant and $\mathrm{NaCl}$. The result of $\mathrm{NaCl}$ test by using the modified gold 15 number method is therefore determined that CMC and PVP-k30 solutions provide the best protection against agglomeration in colloidal nanogold. Of these, CMC is the safer choice as it presents no harm to the human body and is even used in some food items to maintain a food-particle suspension. This study uses the UV-Vis to observe the absorption of the different $\mathrm{pH}$ value. The results 


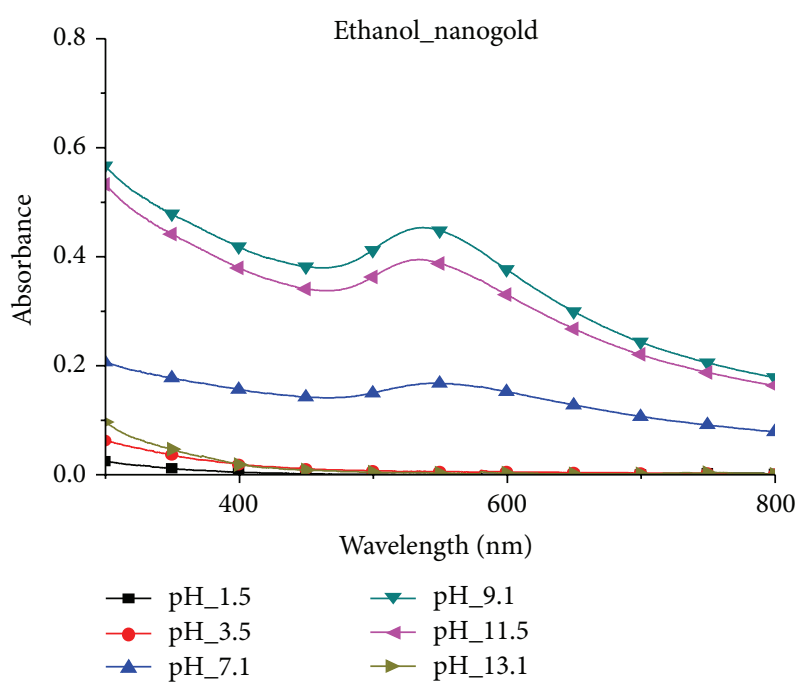

(a)

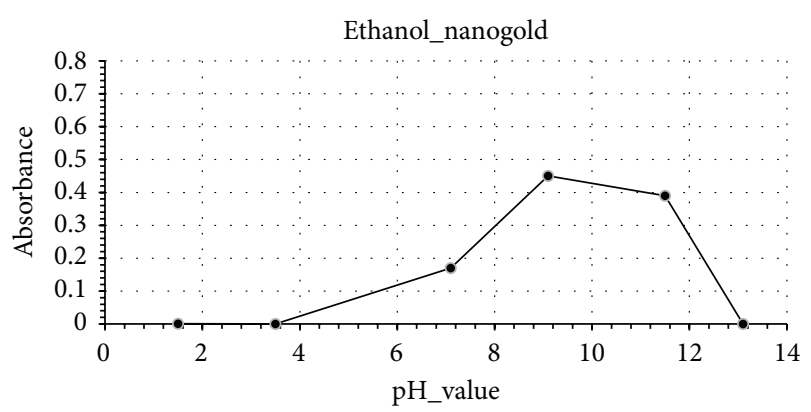

(b)

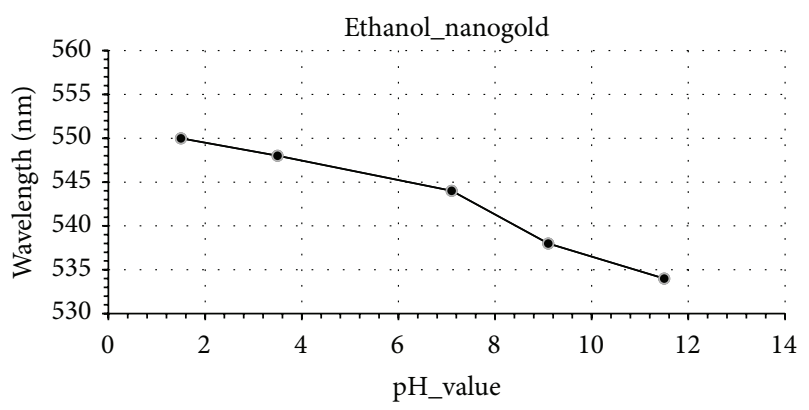

(c)

FIGURE 5: (a) SPR, (b) absorbance, and (c) wavelength versus pH of ethanol nanogold.

show that, in acid liquid, the wavelength of colloidal nanogold is longer, which is red shifting. The absorption of UV-Vis is lower. Also, the Debye Length is shorter. These cause the nanogold particles to assemble and precipitate. In alkalic liquid, the wavelength of colloidal nanogold is shorter, which is blue shifting. The absorption of UV-Vis is higher. Also, the Debye Length is longer. These cause the nanogold particles to disperse. In addition, the result of $\mathrm{pH}$ test shows that a higher $\mathrm{pH}$ will cause the optical property of AuNPs to have a blue shift of the peak wavelength, indicating that the suspension capacity can be improved when the gold nanoparticles are in an alkalic environment. The results of this study can be a reference of nanogold applying in biomedical science.

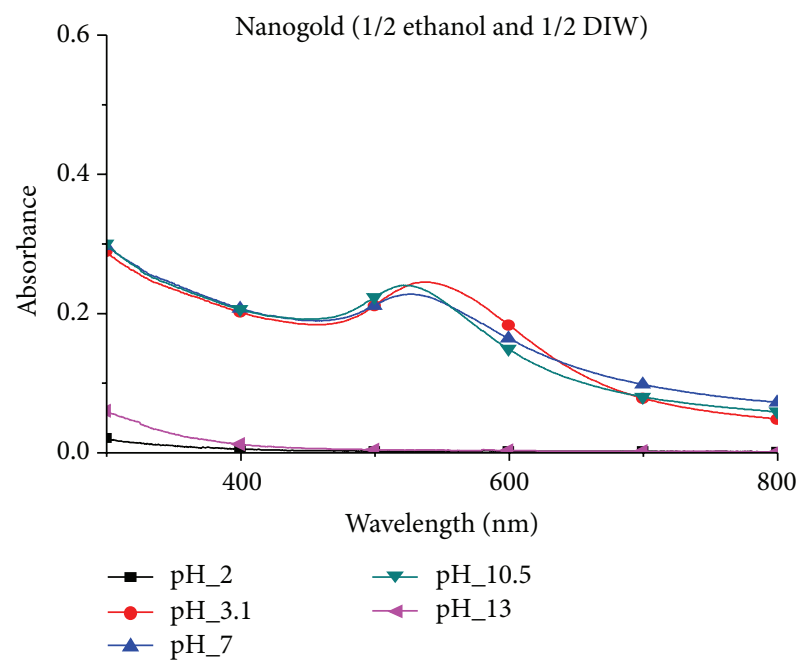

(a)

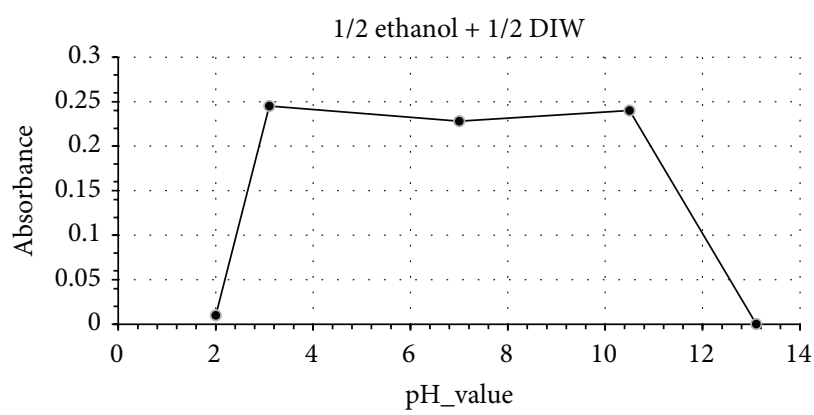

(b)

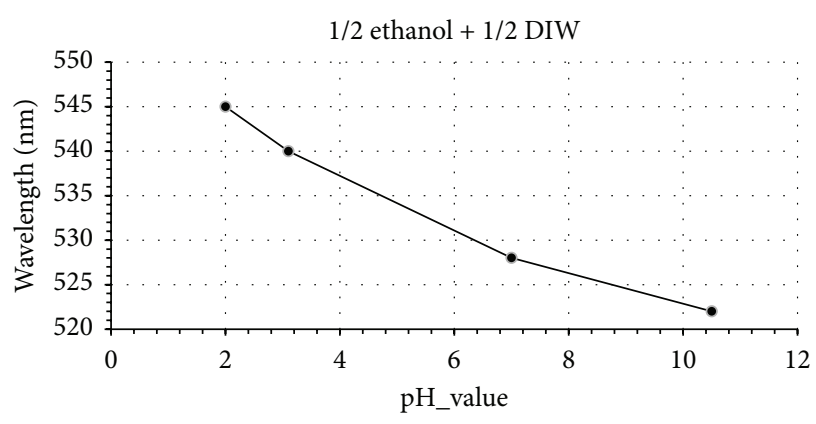

(c)

FIgURE 6: (a) SPR, (b) absorbance, and (c) wavelength versus $\mathrm{pH}$ of ethanol nanogold (1/2 ethanol +1/2 DIW).

\section{Conflict of Interests}

The authors declare that there is no conflict of interests regarding the publication of this paper.

\section{References}

[1] A. Lazcano and J. L. Bada, "The 1953 Stanley L. Miller experiment: fifty years of prebiotic organic chemistry," Origins of Life and Evolution of the Biosphere, vol. 33, no. 3, pp. 235-242, 2003.

[2] K.-H. Tseng, H.-L. Lee, D.-C. Tien, Y.-L. Tang, and Y.-S. Kao, "A study of antibioactivity of nanosilver colloid and silver ion solution," Advances in Materials Science and Engineering, vol. 2014, Article ID 371483, 6 pages, 2014. 
[3] K.-H. Tseng and J.-C. Huang, "Pulsed spark-discharge assisted synthesis of colloidal gold nanoparticles in ethanol," Journal of Nanoparticle Research, vol. 13, no. 7, pp. 2963-2972, 2011.

[4] K.-H. Tseng, J.-C. Huang, C.-Y. Liao, D.-C. Tien, and T.-T. Tsung, "Preparation of gold ethanol colloid by the arc discharge method," Journal of Alloys and Compounds, vol. 472, no. 1-2, pp. 446-450, 2009.

[5] K.-H. Tseng, C.-Y. Liao, J.-C. Huang, D.-C. Tien, and T.-T. Tsung, "Characterization of gold nanoparticles in organic or inorganic medium (ethanol/water) fabricated by spark discharge method," Materials Letters, vol. 62, no. 19, pp. 3341-3344, 2008.

[6] H. Kawaguchi, "The determination of temperature of light sources for spectroscopic analysis from spectra," Spectroscopic Research, vol. 13, no. 1, pp. 1-6, 1964.

[7] W. Natsu, M. Shimoyamada, and M. Kunieda, "Study on expansion process of EDM arc plasma," JSME International Journal, Series C: Mechanical Systems, Machine Elements and Manufacturing, vol. 49, no. 2, pp. 600-605, 2006.

[8] N. Moriyama, J. Sekikawa, and T. Kubono, "Spectroscopic temperature measurement of breaking arcs near cathode and anode surfaces of copper contacts," IEICE Transactions on Electronics, vol. 89, no. 8, pp. 1141-1146, 2006.

[9] H. Gay-Lussac, "Ueber den Cassius'schen Goldpurpur," Annalen der Physik, vol. 101, no. 8, pp. 629-630, 1832.

[10] J. J. Berzelius, “Ueber den Cassius' schen Goldpurpur," Annalen der Physik und Chemie, vol. 98, no. 6, pp. 306-308, 1831.

[11] V. R. Reddy, "Gold nanoparticles: synthesis and applications," Synlett, no. 11, pp. 1791-1792, 2006.

[12] M. Faraday, "The bakerian lecture: experimental relations of gold (and other metals) to light," Philosophical Transactions of the Royal Society of London, vol. 147, no. 0, pp. 145-181, 1857.

[13] V. Sharma, K. Park, and M. Srinivasarao, "Colloidal dispersion of gold nanorods: historical background, optical properties, seed-mediated synthesis, shape separation and self-assembly," Materials Science and Engineering R: Reports, vol. 65, no. 1-3, pp. 1-38, 2009.

[14] B. Papaconstantinou, "The protective action of soaps on zsigmondy's gold sols," The Journal of Physical Chemistry, vol. 29, no. 3, pp. 319-322, 1924.

[15] R. Zsigmondy, "Die hochrothe Goldlösung als Reagens auf Colloide," Zeitschrift für Analytische Chemie, vol. 40, no. 11, pp. 697-719, 1901.

[16] E. C. Dreaden, M. A. MacKey, X. Huang, B. Kang, and M. A. El-Sayed, "Beating cancer in multiple ways using nanogold," Chemical Society Reviews, vol. 40, no. 7, pp. 3391-3404, 2011.

[17] Y. Li, H. J. Schluesener, and S. Xu, "Gold nanoparticle-based biosensors," Gold Bulletin, vol. 43, no. 1, pp. 29-41, 2010.

[18] M.-C. Daniel and D. Astruc, "Gold nanoparticles: assembly, supramolecular chemistry, quantum-size-related properties, and applications toward biology, catalysis, and nanotechnology," Chemical Reviews, vol. 104, no. 1, pp. 293-346, 2004. 

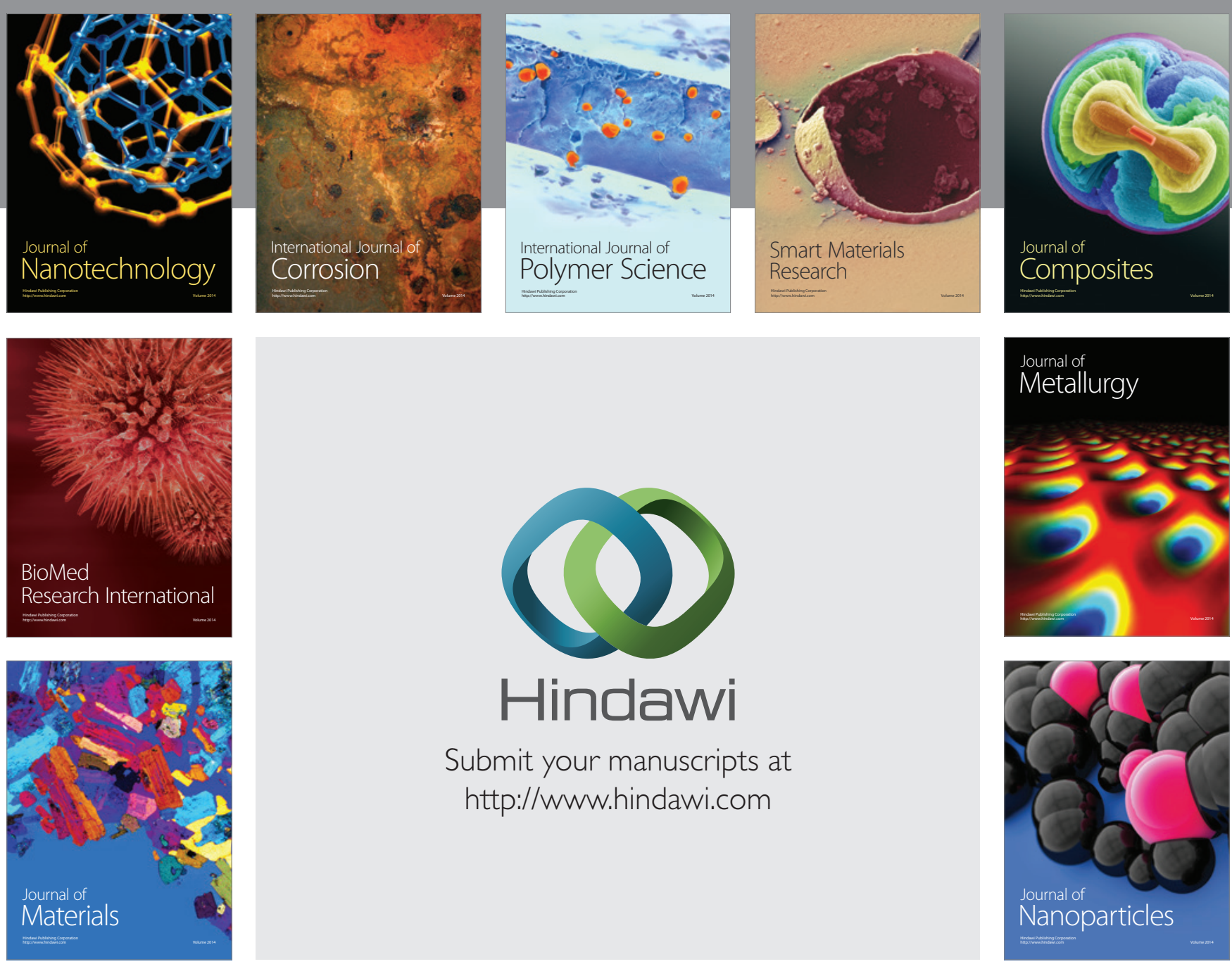

Submit your manuscripts at http://www.hindawi.com
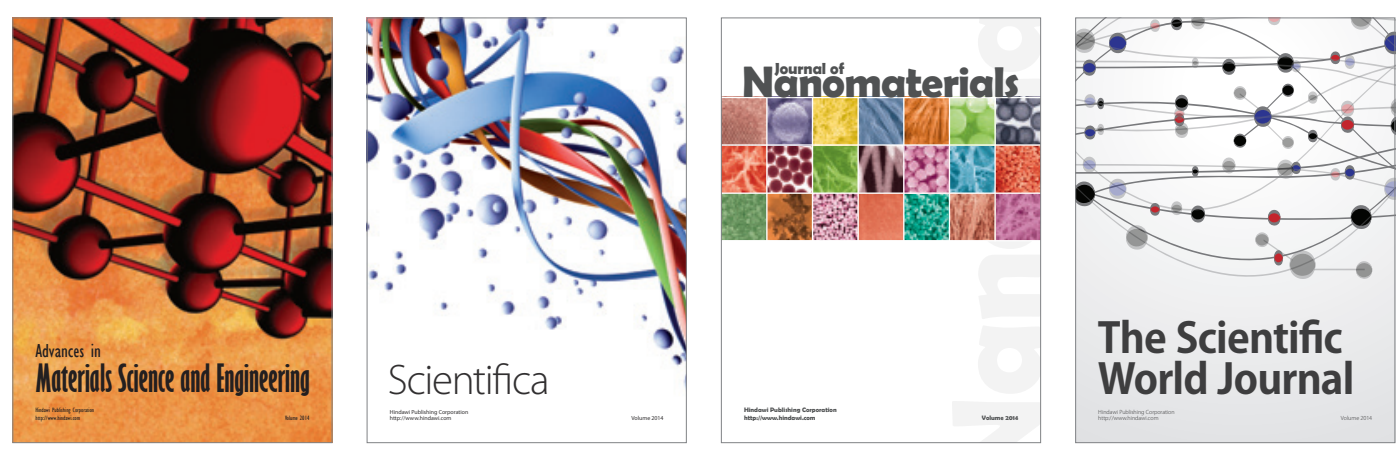

\section{The Scientific World Journal}
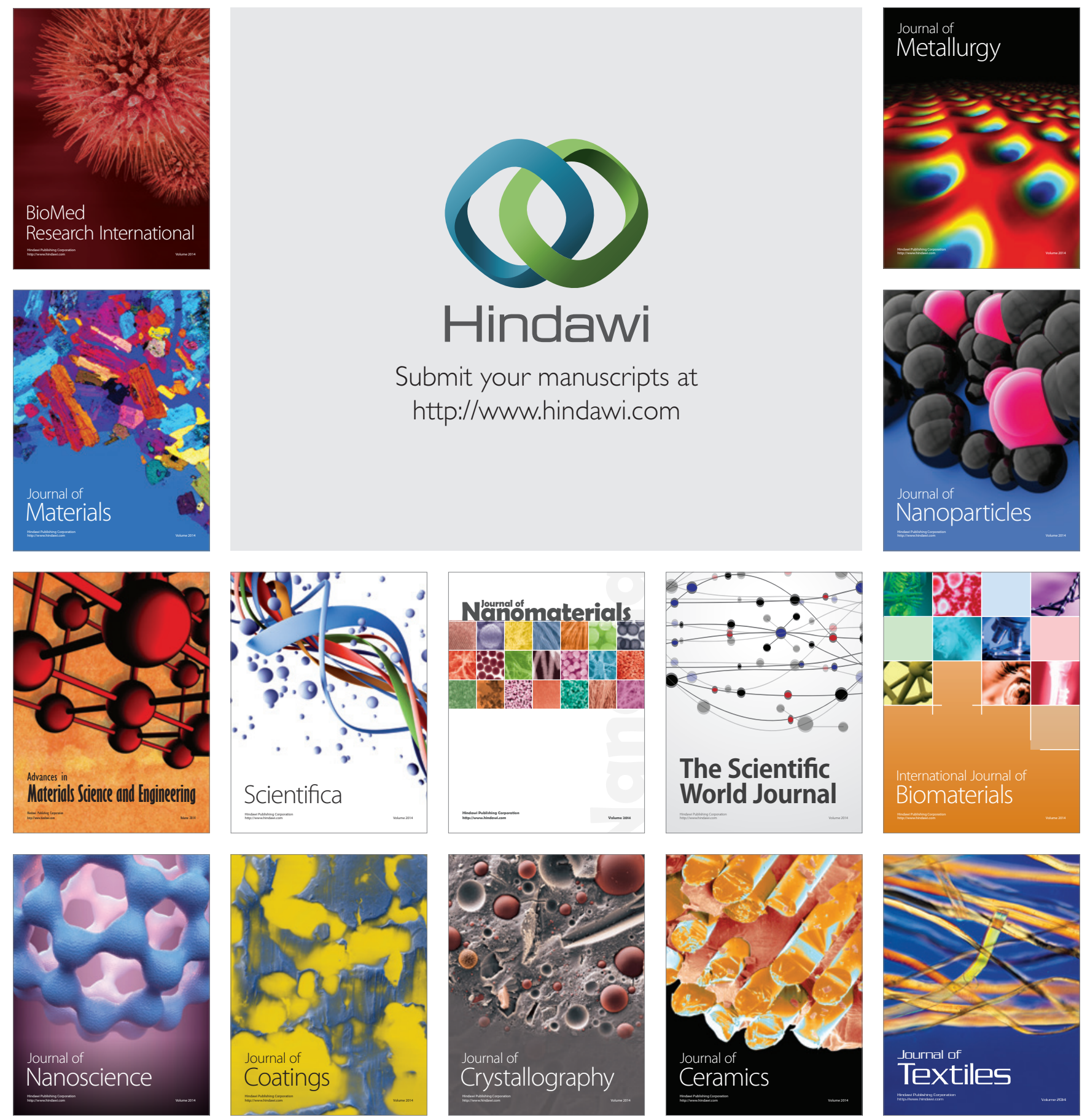